Discussion Paper No. 947

\title{
FAIR REALLOCATION IN ECONOMIES WITH SINGLE-PEAKED PREFERENCES
}

\author{
Kazuhiko Hashimoto \\ Takuma Wakayama
}

September 2015

The Institute of Social and Economic Research Osaka University

6-1 Mihogaoka, Ibaraki, Osaka 567-0047, Japan 


\title{
Fair Reallocation in Economies with Single-Peaked Preferences
}

\author{
Kazuhiko Hashimoto* Takuma Wakayama ${ }^{\dagger}$
}

September 2, 2015

\begin{abstract}
We consider the problem of fairly reallocating the individual endowments of a perfectly divisible good among agents with single-peaked preferences. We provide a new concept of fairness, called position-wise envy-freeness, that is compatible with individual rationality. This new concept requires that each demander (i.e., agent whose most preferred amount is strictly greater than his endowment) should not envy another demander who does not receive his endowment and that each supplier (i.e., agent whose most preferred amount is strictly less than his endowment) should not envy another supplier who does not receive his endowment. We establish that a rule is efficient, individually rational, strategy-proof, and position-wise envy-free if and only if it is the "gradual uniform rule," which is an extension of the well-known uniform rule.

Keywords: Envy-freeness; Individual rationality; Uniform rule; Single-peaked preferences; Strategy-proofness.
\end{abstract}

JEL codes: D71; D63.

*Institute of Social and Economic Research, Osaka University 6-1 Mihogaoka, Ibaraki, Osaka 567-0047, JAPAN; k-hashimoto@iser.osaka-u.ac.jp

${ }^{\dagger}$ Faculty of Economics, Ryukoku University, 67 Tsukamoto-cho, Fukakusa, Fushimi-ku, Kyoto 612-8577, JAPAN; wakayama@econ.ryukoku.ac.jp 


\section{Introduction}

We consider the problem of fairly reallocating the individual endowments of a perfectly divisible good among agents with single-peaked preferences. ${ }^{1}$ This situation may occur particularly when the existing allocation is unsatisfactory owing to changes in preferences over time. ${ }^{2}$

Envy-freeness is the most standard concept of fairness in the literature. It states that every agent prefers his assignment to everybody else's. However, this concept is quite demanding in our setting, because the set of individually rational (no agent prefers his endowment to his own assignment) and envy-free allocations might be empty. This motivates us to search for fairness concepts that are compatible with individual rationality.

We propose a new notion of fairness called position-wise envy-freeness. In our setting, we categorize "traders" (i.e., agents whose most preferred amounts are not equal to their endowments) into two positions: an agent is a "demander" ("supplier") if his endowment is strictly less (greater) than his most preferred amount. The notion of position-wise envy-freeness then states that each demander (supplier) prefers his own assignment to another demander's (supplier's) assignment that is not equal to his endowment. That is, we weaken envy-freeness by only requiring each agent not to envy another agent who is in the same position and does not receive his endowment. The existence of individually rational and position-wise envy-free allocations, of course, is guaranteed.

We next construct a position-wise envy-free and individually rational rule. We call it the "gradual uniform rule." This rule is an extension of the uniform rule (Benassy, 1982), which is the best-known rule in this problem. Furthermore, we establish that the gradual uniform rule is the only one satisfying efficiency (no Pareto improvement is possible) and strategy-proofness (no one can gain by preference misrepresentation) in addition to individual rationality and position-wise envy-freeness.

There is another way to extend the notion of envy-freeness to the case of economies with individual endowments, which is to define envy-freeness in terms of changes in allocations rather than in terms of final allocations. This concept is called envyfreeness on net trade, which requires that no agent prefers another agent's net trade

\footnotetext{
${ }^{1}$ This "reallocation problem," first analyzed by Klaus et al. (1997, 1998a, 1998b), is a natural extension of the problem of fairly allocating a social endowment of a perfectly divisible good among agents having single-peaked preferences (Sprumont, 1991). For comprehensive surveys of the literature on private good economies in which agents have single-peaked preferences, see Klaus (1998) and Thomson (2014).

${ }^{2}$ See Klaus et al. $(1997,1998 \mathrm{a}, 1998 \mathrm{~b})$ and Klaus (1998) for interpretations of this problem.
} 
to his own. ${ }^{3}$ It is also compatible with individual rationality. Klaus et al. (1997, 1998a, 1998b) apply it to our setting and construct an envy-free on net trade rule called "uniform reallocation rule." The uniform reallocation rule, which is another extension of the uniform rule, is the only one satisfying efficiency, strategy-proofness, and envy-freeness on net trade (Klaus et al., 1998b). However, the uniform reallocation rule is not position-wise envy-free. This suggests that whenever we insist on fairness criteria for final allocations rather than for changes in allocations, the gradual uniform rule is more favorable than the uniform reallocation rule.

In Section 2, we set up the model and introduce basic properties of allocations and rules. In Section 3, we spell out a new concept of fairness. In Section 4, we introduce the three main rules and show the main result.

\section{Preliminaries}

Let $N=\{1, \ldots, n\}$ be the set of agents. There is one perfectly divisible good. Each agent $i \in N$ owns an individual endowment $e_{i} \in \mathbb{R}_{+}$of the good. Let $e \equiv$ $\left(e_{1}, \ldots, e_{n}\right) \in \mathbb{R}_{+}^{n}$ be the profile of individual endowments, and let $E \equiv \sum_{i \in N} e_{i}$. Each agent $i \in N$ has a single-peaked preference relation $R_{i}$ on $[0, E]$ : there is a point $p\left(R_{i}\right) \in[0, E]$ such that for each pair $\left\{x_{i}, y_{i}\right\} \subset[0, E]$, if either $y_{i}<x_{i} \leq p\left(R_{i}\right)$ or $p\left(R_{i}\right) \leq x_{i}<y_{i}$, then $x_{i} P_{i} y_{i}$, where $P_{i}$ is the asymmetric part of $R_{i}$. The point $p\left(R_{i}\right)$ is called the peak of $R_{i}$. Let $p(R) \equiv\left(p\left(R_{1}\right), \ldots, p\left(R_{n}\right)\right)$ be the profile of peaks. We denote the set of all single-peaked preferences defined on $[0, E]$ by $\mathscr{R}$. An element $R \equiv\left(R_{1}, \ldots, R_{n}\right)$ of $\mathscr{R}^{n}$ is called a preference profile. Given $R \in \mathscr{R}^{n}$ and $i \in N$, let $R_{-i} \equiv\left(R_{j}\right)_{j \neq i}$. The set of feasible allocations is

$$
X \equiv\left\{\left(x_{1}, \ldots, x_{n}\right) \in \mathbb{R}_{+}^{n}: \sum_{i \in N} x_{i}=E\right\}
$$

A rule is a function $f: \mathscr{R}^{n} \rightarrow X$ assigning to each preference profile $R \in \mathscr{R}^{n}$ a feasible allocation $f(R) \equiv\left(f_{1}(R), \ldots, f_{n}(R)\right) \in X$, where $f_{i}(R)$ means agent $i$ 's assignment at $R$.

The following properties of allocations and rules are standard in the literature.

- Efficiency: An allocation $x \in X$ is efficient for $R \in \mathscr{R}^{n}$ if there is no $x^{\prime} \in X$ such that for each $i \in N, x_{i}^{\prime} R_{i} x_{i}$, and for some $j \in N, x_{j}^{\prime} P_{j} x_{j}$. A rule $f$ is

\footnotetext{
${ }^{3}$ The notion of envy-freeness in terms of changes in allocations is first formulated by Schmeidler and Vind (1972) in the context of pure exchange economies.
} 
efficient if for each $R \in \mathscr{R}^{n}, f(R)$ is efficient for $R$.

- Individual rationality: An allocation $x \in X$ is individually rational for $R \in \mathscr{R}^{n}$ if for each $i \in N, x_{i} R_{i} e_{i}$. A rule $f$ is individually rational if for each $R \in \mathscr{R}^{n}, f(R)$ is individually rational for $R$.

- Strategy-proofness: For each $R \in \mathscr{R}^{n}$, each $i \in N$, and each $R_{i}^{\prime} \in \mathscr{R}$, $f_{i}(R) R_{i} f_{i}\left(R_{i}^{\prime}, R_{-i}\right)$.

Efficiency requires that there be no other feasible allocation such that someone can be made better off without anyone else being made worse off. Individual rationality requires that no agent prefers his individual endowment to his own assignment. Strategy-proofness requires that no agent can ever benefit from misrepresenting his preferences.

\section{Position-Wise Envy-Freeness}

The following property of fairness is central to the mechanism design literature.

- Envy-freeness: An allocation $x \in X$ is envy-free for $R \in \mathscr{R}^{n}$ if for each pair $\{i, j\} \subseteq N, x_{i} R_{i} x_{j}$. A rule $f$ is envy-free if for each $R \in \mathscr{R}^{n}, f(R)$ is envy-free for $R$.

Envy-freeness, which is first introduced by Foley (1967), requires that no agent prefers another agent's assignment to his own. Unfortunately, in our setting, the set of individually rational and envy-free allocations might be empty. This observation motivates us to seek meaningful notions of fairness that are compatible with individual rationality.

We now introduce a new concept of fairness that is compatible with individual rationality. In doing so, we call an agent a trader if his endowment is not equal to his peak. We now categorize traders into two "positions": an agent is a demander (supplier) if his endowment is strictly less (greater) than his peak. Given $R \in \mathscr{R}^{n}$, let $N^{d}(R) \equiv\left\{i \in N: p\left(R_{i}\right)>e_{i}\right\}$ and $N^{s}(R) \equiv\left\{i \in N: p\left(R_{i}\right)<e_{i}\right\}$ be the sets of demanders and suppliers at $R$, respectively.

- Position-wise envy-freeness: An allocation $x \in X$ is position-wise envyfree for $R \in \mathscr{R}^{n}$ if for each pair $\{i, j\} \subseteq N$, if $x_{j} \neq e_{j}$ and either $\{i, j\} \subseteq N^{d}(R)$ or $\{i, j\} \subseteq N^{s}(R)$, then $x_{i} R_{i} x_{j}$. A rule $f$ is position-wise envy-free if for each $R \in \mathscr{R}^{n}, f(R)$ is position-wise envy-free for $R$. 
Position-wise envy-freeness states that each demander (supplier) prefers his own assignment to another demander's (supplier's) assignment that is not equal to his individual endowment. Hence, we weaken the notion of envy-freeness by allowing agents to envy (i) agents not in the same position and (ii) agents receiving their endowments.

It should be noted that an individually rational and position-wise envy-free allocation always exists. A typical example of such an allocation is the profile of individual endowments.

Remark 1. Klaus et al. (1998b) consider the following notion of fairness that is compatible with individual rationality:

- Envy-freeness on net trade: For each $R \in \mathscr{R}^{n}$ and each pair $\{i, j\} \subseteq N$, $f_{i}(R) R_{i} \max \left\{e_{i}+\left(f_{j}(R)-e_{j}\right), 0\right\}$.

It requires that no agent prefers another agent's net trade to his own. Although both this notion and our notion incorporate individual endowments into envy-freeness, they are based on significantly different concepts: the former is related to fairness criteria for final allocations, and the latter, to fairness criteria for changes in allocations. ${ }^{4}$

\section{Results}

The following rule is the best-known one in the social endowment setting. ${ }^{5}$

Uniform rule, $\boldsymbol{U}$ : For each $R \in \mathscr{R}^{n}$ and each $i \in N$,

$$
U_{i}(R)= \begin{cases}\min \left\{p\left(R_{i}\right), \lambda\right\} & \text { if } \sum_{j \in N} p\left(R_{j}\right) \geq E \\ \max \left\{p\left(R_{i}\right), \lambda\right\} & \text { if } \sum_{j \in N} p\left(R_{j}\right) \leq E\end{cases}
$$

where $\lambda \in \mathbb{R}_{+}$solves $\sum_{j \in N} U_{j}(R)=E$.

\footnotetext{
${ }^{4}$ As mentioned in Introduction, envy-freeness on net trade is based on the idea of Schmeidler and Vind (1972).

${ }^{5}$ The uniform rule is introduced by Benassy (1982). Sprumont (1991) first provides an axiomatic characterization of the uniform rule in the social endowment setting. Subsequently, there have been many studies on characterizing the uniform rule in the social endowment setting. See, for example, Ching (1994), Thomson (1994a, 1994b, 1995, 1997), Chun (2006), Serizawa (2006), Mizobuchi and Serizawa (2006), Sakai and Wakayama (2012), and Wakayama (2015). See also Thomson (2014) for a survey of the characterizations of the uniform rule. Sönmez (1994) and Barberà et al. (1997) describe an algorithm that computes the allocation of the uniform rule.
} 
The uniform rule is efficient, strategy-proof, and envy-free. Moreover, it is the only rule satisfying these three properties (Sprumont, 1991). However, this rule violates individual rationality. ${ }^{6}$ In this sense, the uniform rule is not suitable for use in our setting.

The following rule extends the uniform rule to account for individual endowments.

Uniform reallocation rule, $\boldsymbol{U}^{r}$ : For each $R \in \mathscr{R}^{n}$ and each $i \in N$,

$$
U_{i}^{r}(R)= \begin{cases}\min \left\{p\left(R_{i}\right), e_{i}+\lambda\right\} & \text { if } \sum_{j \in N} p\left(R_{j}\right) \geq E \\ \max \left\{p\left(R_{i}\right), e_{i}-\lambda\right\} & \text { if } \sum_{j \in N} p\left(R_{j}\right) \leq E\end{cases}
$$

where $\lambda \in \mathbb{R}_{+}$solves $\sum_{j \in N} U_{j}^{r}(R)=E$.

The uniform reallocation rule is the most studied in our setting. ${ }^{7}$ It is well known that the uniform reallocation rule is the only one satisfying efficiency, strategyproofness, and envy-freeness on net trade (Klaus et al., 1998b). Furthermore, it is individually rational. However, as we show below, this rule violates position-wise envy-freeness.

Proposition 1. The uniform reallocation rule is not position-wise envy-free.

Proof. Without loss of generality, we assume $n=3$. Let $e=(1,5,10)$ and $R \in \mathscr{R}^{3}$ be such that $p(R)=(6,15,8)$. Then, $U^{r}(R)=(2,6,8)$. Hence, $\{1,2\} \subseteq N^{d}(R)$ and $U_{2}^{r}(R)>e_{2}$, but $U_{2}^{r}(R)=6 P_{1} 2=U_{1}^{r}(R)$. This means that the uniform reallocation rule is not position-wise envy-free.

We extend the uniform rule to satisfy both individual rationality and positionwise envy-freeness. ${ }^{8}$

Gradual uniform rule, $\boldsymbol{G}$ : For each $R \in \mathscr{R}^{n}$ and each $i \in N$,

$$
G_{i}(R)= \begin{cases}\min \left\{p\left(R_{i}\right), \max \left\{e_{i}, \lambda\right\}\right\} & \text { if } \sum_{j \in N} p\left(R_{j}\right) \geq E, \\ \max \left\{p\left(R_{i}\right), \min \left\{e_{i}, \lambda\right\}\right\} & \text { if } \sum_{j \in N} p\left(R_{j}\right) \leq E,\end{cases}
$$

where $\lambda \in \mathbb{R}_{+}$solves $\sum_{j \in N} G_{j}(R)=E$.

\footnotetext{
${ }^{6}$ As mentioned above, envy-freeness is incompatible with individual rationality.

${ }^{7}$ See Klaus et al. (1997, 1998a, 1998b), Klaus (1998, 2001), Moreno (2002), and Bonifacio (2015).

${ }^{8} \mathrm{~A}$ similar functional form appears in the social endowment setting under constraints. See Bergantiños et al. (2015).
} 
The gradual uniform rule $G$ is an extension of the uniform rule $U$ in the sense that if for each pair $\{i, j\} \subseteq N, e_{i}=e_{j}$, then $U(R)=G(R)$.

The following example demonstrates the computation of the gradual uniform allocation for the case where the sum of agents' peaks is strictly greater than the sum of individual endowments.

Example 1. Let $N=\{1,2,3,4\}$ and $e=(1,7,3,10)$. Let $R \in \mathscr{R}^{4}$ be such that $p(R)=(7,13,4,4)$. Therefore, the sum of the peaks is strictly greater than the sum of the individual endowments. Then, the gradual uniform allocation of this problem can be determined as follows. Agent 4 receives his peak. We now have to divide the amount agent 4 supplies (i.e., the six units of the good) among the demanders. We increase the amounts of the demanders until we have assigned the amount agent 4 supplies as follows. We first increase the amount of the demander with the smallest individual endowment (i.e., agent 1) until it reaches either his peak or the second smallest individual endowment among the demanders (i.e., agent 3's individual endowment). We then obtain "temporary" allocation $(3,7,3,4)$. There remain the four units of the good. We next increase the amounts of agents 1 and 3 equally until one of them reaches either his peak or the third smallest individual endowment among demanders (i.e., agent 2's individual endowment). We then obtain "temporary" allocation $(4,7,4,4)$. Now, agent 3 receives his peak. We still have to allocate the two unit of the good. Again, we increase the amount of agent 1 until it reaches either his peak or the third smallest individual endowment among demanders. We then obtain allocation $(6,7,4,4)$ and complete this process. Note that this example yields $\lambda=6$. We note further that allocation $(6,7,4,4)$ is individually rational and position-wise envy-free.

From Example 1, we observe that there exist differences between the gradual uniform rule and the uniform reallocation rule. According to the latter rule, we obtain allocation $(3.5,9.5,4,4)$. However, this allocation is not position-wise envyfree, because both agent 1 and agent 3 are demanders, and agent 1 envies agent 3 who does not receive his endowment. In contrast, the gradual uniform rule selects a position-wise envy-free allocation. In fact, for every preference profile, the gradual uniform rule selects a position-wise envy-free allocation.

Proposition 2. The gradual uniform rule is position-wise envy-free.

Proof. Let $R \in \mathscr{R}^{n}$. In order to prove position-wise envy-freeness of $G$, we have to show that for each pair $\{i, j\} \subseteq N$, if $G_{j}(R) \neq e_{j}$ and either $\{i, j\} \subseteq N^{d}(R)$ or 
$\{i, j\} \subseteq N^{s}(R)$, then

$$
G_{i}(R) R_{i} G_{j}(R)
$$

Let $i \in N$. Consider the case $\sum_{j \in N} p\left(R_{j}\right) \geq E$ (the other case is similar and we omit the details). We distinguish two cases.

Case 1: $\boldsymbol{i} \notin \boldsymbol{N}^{\boldsymbol{d}}(\boldsymbol{R})$. Then, $G_{i}(R)=p\left(R_{i}\right)$. This implies (1).

Case 2: $\boldsymbol{i} \in \boldsymbol{N}^{d}(\boldsymbol{R})$. Let $j \in N^{d}(R)$ be such that $G_{j}(R) \neq e_{j}$. Then, $e_{j}<\lambda$. Otherwise, $G_{j}(R) \equiv \min \left\{p\left(R_{j}\right), \max \left\{e_{j}, \lambda\right\}\right\}=\min \left\{p\left(R_{j}\right), e_{j}\right\}$. Since $p\left(R_{j}\right)>e_{j}$, we have $G_{j}(R)=e_{j}$, a contradiction. Therefore,

$$
G_{j}(R) \equiv \min \left\{p\left(R_{j}\right), \max \left\{e_{j}, \lambda\right\}\right\}=\min \left\{p\left(R_{j}\right), \lambda\right\}
$$

By the definition of $G$, there are two subcases.

Subcase 2.1: $\boldsymbol{p}\left(\boldsymbol{R}_{\boldsymbol{i}}\right) \leq \max \left\{\boldsymbol{e}_{\boldsymbol{i}}, \boldsymbol{\lambda}\right\}$. Then, $G_{i}(R)=p\left(R_{i}\right)$. Hence, (1) is trivially true.

Subcase 2.2: $\boldsymbol{p}\left(\boldsymbol{R}_{\boldsymbol{i}}\right)>\max \left\{\boldsymbol{e}_{\boldsymbol{i}}, \boldsymbol{\lambda}\right\}$. By (2),

$$
G_{j}(R)=\min \left\{p\left(R_{j}\right), \lambda\right\} \leq \lambda \leq \max \left\{e_{i}, \lambda\right\}<p\left(R_{i}\right)
$$

Since $G_{i}(R)=\max \left\{e_{i}, \lambda\right\}$, this implies $(1)$.

The gradual uniform rule satisfies not only individual rationality and positionwise envy-freeness but also efficiency and strategy-proofness. Furthermore, we show that it is the only rule satisfying these properties.

Theorem 1. The gradual uniform rule is the only rule that satisfies efficiency, individual rationality, strategy-proofness, and position-wise envy-freeness.

Proof. It is easy to see that the gradual uniform rule $G$ is efficient, individually rational, and strategy-proof. By Proposition 2, $G$ is position-wise envy-free.

To prove the remaining part of the theorem, let $f$ be a rule satisfying the four properties. Let $R \in \mathscr{R}^{n}$. We show that for each $i \in N, f_{i}(R)=G_{i}(R)$. Consider the case $\sum_{j \in N} p\left(R_{j}\right) \geq E$ (the other case is similar and we omit the details).

Claim 1. For each $i \notin N^{d}(R), f_{i}(R)=p\left(R_{i}\right)=G_{i}(R)$.

Proof of Claim 1. Let $i \notin N^{d}(R)$. Then, $p\left(R_{i}\right) \leq e_{i}$. By the definition of $G, p\left(R_{i}\right)=$ $G_{i}(R)$ is obvious. We prove only $f_{i}(R)=p\left(R_{i}\right)$. Suppose, by contradiction, that 
$f_{i}(R) \neq p\left(R_{i}\right)$. It then follows from efficiency that $f_{i}(R)<p\left(R_{i}\right)$. Since $p\left(R_{i}\right) \leq e_{i}$, we can take $R_{i}^{\prime} \in \mathscr{R}$ such that $p\left(R_{i}^{\prime}\right)=p\left(R_{i}\right)$ and $e_{i} P_{i}^{\prime} f_{i}(R)$. By individual rationality and efficiency, $f_{i}(R)<f_{i}\left(R_{i}^{\prime}, R_{-i}\right) \leq p\left(R_{i}\right)$. Then, $f_{i}\left(R_{i}^{\prime}, R_{-i}\right) P_{i} f_{i}(R)$, which contradicts strategy-proofness. Hence, $f_{i}(R)=p\left(R_{i}\right)$.

If for each $i \in N^{d}(R), f_{i}(R) \geq G_{i}(R)$, then Claim 1 and feasibility together imply that for each $i \in N^{d}(R), f_{i}(R)=G_{i}(R)$. This, together with Claim 1, yields that for each $i \in N, f_{i}(R)=G_{i}(R)$. Therefore, in what follows, suppose that there is $j \in N^{d}(R)$ with $f_{j}(R)<G_{j}(R)$. Without loss of generality, we assume

$$
f_{1}(R)=\min \left\{f_{j}(R): f_{j}(R)<G_{j}(R)\right\}
$$

Claim 1 ensures that $1 \in N^{d}(R)$, that is,

$$
p\left(R_{1}\right)>e_{1}
$$

Claim 2. $f_{1}(R)<\lambda$.

Proof of Claim 2. Suppose, by contradiction, that $f_{1}(R) \geq \lambda$. By $(3), f_{1}(R)<$ $G_{1}(R) \equiv \min \left\{p\left(R_{1}\right), \max \left\{e_{1}, \lambda\right\}\right\}$. It then follows that $f_{1}(R)<\max \left\{e_{1}, \lambda\right\}$. If $\max \left\{e_{1}, \lambda\right\}=\lambda$, then $\lambda>f_{1}(R) \geq \lambda$, a contradiction. If $\max \left\{e_{1}, \lambda\right\}=e_{1}$, then (4) implies that $f_{1}(R)<e_{1}<p\left(R_{1}\right)$, which contradicts individual rationality. Hence, $f_{1}(R)<\lambda$.

Claim 3. For each $i \in N^{d}(R)$, if $f_{1}(R)>f_{i}(R)$, then $f_{i}(R)=p\left(R_{i}\right)$.

Proof of Claim 3. Suppose, by contradiction, that there is $i \in N^{d}(R)$ such that $f_{1}(R)>f_{i}(R)$ and $f_{i}(R) \neq p\left(R_{i}\right)$. Then, (3) implies that $f_{i}(R) \geq G_{i}(R)$. Thus, by Claim 2,

$$
\lambda>f_{1}(R)>f_{i}(R) \geq G_{i}(R) \equiv \min \left\{p\left(R_{i}\right), \max \left\{e_{i}, \lambda\right\}\right\}
$$

If $\min \left\{p\left(R_{i}\right), \max \left\{e_{i}, \lambda\right\}\right\}=p\left(R_{i}\right)$, then $f_{i}(R) \geq p\left(R_{i}\right)$. Since $f_{i}(R) \neq p\left(R_{i}\right)$, $f_{i}(R)>p\left(R_{i}\right)$, which contradicts efficiency. If $\min \left\{p\left(R_{i}\right), \max \left\{e_{i}, \lambda\right\}\right\}=\max \left\{e_{i}, \lambda\right\}$, then $f_{i}(R) \geq \max \left\{e_{i}, \lambda\right\} \geq \lambda$. This, together with (5), implies that $\lambda>\lambda$, a contradiction.

Claim 4. There is $h \in N^{d}(R)$ such that $f_{h}(R)>e_{h}$ and $f_{h}(R)>f_{1}(R)$. 
Proof of Claim 4. Note that by $(3), f_{1}(R)<G_{1}(R)$. Thus, by feasibility, there is $h \in N$ such that $f_{h}(R)>G_{h}(R) \equiv \min \left\{p\left(R_{h}\right), \max \left\{e_{h}, \lambda\right\}\right\}$. By Claim $1, h \in$ $N^{d}(R)$. We also assert that $\min \left\{p\left(R_{h}\right), \max \left\{e_{h}, \lambda\right\}\right\}=\max \left\{e_{h}, \lambda\right\}$, that is, $f_{h}(R)>$ $\max \left\{e_{h}, \lambda\right\}$; otherwise, $f_{h}(R)>p\left(R_{h}\right)$, which contradicts efficiency. Hence, $f_{h}(R)>$ $e_{h}$. It also follows from Claim 2 that $f_{h}(R)>\max \left\{e_{h}, \lambda\right\} \geq \lambda>f_{1}(R)$.

Notice that since $G$ is efficient, (3) implies that $E \geq p\left(R_{1}\right) \geq G_{1}(R)>f_{1}(R)$. Therefore, we can take $\hat{R}_{1} \in \mathscr{R}$ such that

$$
p\left(\hat{R}_{1}\right)=p\left(R_{1}\right) \text { and } E \hat{P}_{1} f_{1}(R) .
$$

Claim 5. For each $i \in N, f_{i}\left(\hat{R}_{1}, R_{-1}\right) \leq f_{i}(R)$.

Proof of Claim 5. Let $i \in N$. We divide the argument into three cases.

Case 1: $\boldsymbol{i}=1$. By efficiency, $f_{1}(R) \leq p\left(R_{1}\right)=p\left(\hat{R}_{1}\right)$ and $f_{1}\left(\hat{R}_{1}, R_{-1}\right) \leq$ $p\left(R_{1}\right)=p\left(\hat{R}_{1}\right)$. If $f_{1}(R)<f_{1}\left(\hat{R}_{1}, R_{-1}\right)$, then $f_{1}\left(\hat{R}_{1}, R_{-1}\right) P_{1} f_{1}(R)$, which contradicts strategy-proofness. If $f_{1}\left(\hat{R}_{1}, R_{-1}\right)<f_{1}(R)$, then $f_{1}(R) \hat{P}_{1} f_{1}\left(\hat{R}_{1}, R_{-1}\right)$, which contradicts strategy-proofness. Hence, $f_{1}\left(\hat{R}_{1}, R_{-1}\right)=f_{1}(R)$.

Case 2: $\boldsymbol{i} \notin \boldsymbol{N}^{\boldsymbol{d}}(\boldsymbol{R})$. By the same way as Claim 1 , we have $f_{i}\left(\hat{R}_{1}, R_{-1}\right)=p\left(R_{i}\right)$. Hence, $f_{i}\left(\hat{R}_{1}, R_{-1}\right)=f_{i}(R)$.

Case 3: $i \in N^{d}(R)$. Then,

$$
p\left(R_{i}\right)>e_{i}
$$

Now, there are three subcases.

Subcase 3.1: $\boldsymbol{f}_{\mathbf{1}}(\boldsymbol{R})>\boldsymbol{f}_{\boldsymbol{i}}(\boldsymbol{R})$. By Claim $3, f_{i}(R)=p\left(R_{i}\right)$. Then, by efficiency, $f_{i}\left(\hat{R}_{1}, R_{-1}\right) \leq p\left(R_{i}\right)=f_{i}(R)$.

Subcase 3.2: $\boldsymbol{f}_{\mathbf{1}}(\boldsymbol{R})=\boldsymbol{f}_{\boldsymbol{i}}(\boldsymbol{R})$. Suppose, by contradiction, that $f_{i}\left(\hat{R}_{1}, R_{-1}\right)>$ $f_{i}(R)$. Then, $f_{i}\left(\hat{R}_{1}, R_{-1}\right) \neq e_{i}$; otherwise, by $(7), p\left(R_{i}\right)>e_{i}=f_{i}\left(\hat{R}_{1}, R_{-1}\right)>f_{i}(R)$, which contradicts individual rationality. By Case $1, f_{i}\left(\hat{R}_{1}, R_{-1}\right)>f_{i}(R)=f_{1}(R)=$ $f_{1}\left(\hat{R}_{1}, R_{-1}\right)$. By efficiency, $p\left(\hat{R}_{1}\right)=p\left(R_{1}\right)>f_{1}(R)=f_{1}\left(\hat{R}_{1}, R_{-1}\right)$. Then, either

(i) $p\left(\hat{R}_{1}\right) \geq f_{i}\left(\hat{R}_{1}, R_{-1}\right)>f_{1}\left(\hat{R}_{1}, R_{-1}\right)$ or

(ii) $E \geq f_{i}\left(\hat{R}_{1}, R_{-1}\right)>p\left(\hat{R}_{1}\right)>f_{1}\left(\hat{R}_{1}, R_{-1}\right)$. 
In either case, by (6),

$$
f_{i}\left(\hat{R}_{1}, R_{-1}\right) \hat{P}_{1} f_{1}\left(\hat{R}_{1}, R_{-1}\right)
$$

Since $f_{i}\left(\hat{R}_{1}, R_{-1}\right) \neq e_{i}$ and $\{1, i\} \subseteq N^{d}\left(\hat{R}_{1}, R_{-1}\right)$, (8) implies a contradiction to position-wise envy-freeness. Hence, $f_{i}\left(\hat{R}_{1}, R_{-1}\right) \leq f_{i}(R)$.

Subcase 3.3: $\boldsymbol{f}_{\mathbf{1}}(\boldsymbol{R})<\boldsymbol{f}_{\boldsymbol{i}}(\boldsymbol{R})$. By position-wise envy-freeness, either

(i) $f_{i}\left(\hat{R}_{1}, R_{-1}\right)=e_{i}$ or

(ii) $f_{i}\left(\hat{R}_{1}, R_{-1}\right) \leq f_{1}\left(\hat{R}_{1}, R_{-1}\right)$.

If (i) holds, then $f_{i}\left(\hat{R}_{1}, R_{-1}\right)=e_{i} \leq f_{i}(R)$; otherwise, by (7), $p\left(R_{i}\right)>e_{i}=$ $f_{i}\left(\hat{R}_{1}, R_{-1}\right)>f_{i}(R)$, which contradicts individual rationality. If (ii) holds, then, by Case $1, f_{i}\left(\hat{R}_{1}, R_{-1}\right) \leq f_{1}\left(\hat{R}_{1}, R_{-1}\right)=f_{1}(R)<f_{i}(R)$. Hence, $f_{i}\left(\hat{R}_{1}, R_{-1}\right) \leq$ $f_{i}(R)$.

Claim 6. There is $h \in N^{d}(R)$ such that $f_{h}\left(\hat{R}_{1}, R_{-1}\right)<f_{h}(R)$.

Proof of Claim 6. By Claim 4, there is $h \in N^{d}(R)$ such that $f_{h}(R)>e_{h}$ and $f_{h}(R)>f_{1}(R)$. Then, we show that $f_{h}\left(\hat{R}_{1}, R_{-1}\right)<f_{h}(R)$. Otherwise, since $f_{1}(R)=$ $f_{1}\left(\hat{R}_{1}, R_{-1}\right)$ (Case 1 of Claim 5$), f_{h}\left(\hat{R}_{1}, R_{-1}\right) \geq f_{h}(R)>f_{1}(R)=f_{1}\left(\hat{R}_{1}, R_{-1}\right)$. It then follows from (6) that

$$
f_{h}\left(\hat{R}_{1}, R_{-1}\right) \hat{P}_{1} f_{1}\left(\hat{R}_{1}, R_{-1}\right)
$$

Since $f_{h}\left(\hat{R}_{1}, R_{-1}\right) \geq f_{h}(R)>e_{h}$ and $\{1, h\} \subseteq N^{d}\left(\hat{R}_{1}, R_{-1}\right)$, (9) implies a contradiction to position-wise envy-freeness.

By Claim 5, for each $i \in N, f_{i}\left(\hat{R}_{1}, R_{-1}\right) \leq f_{i}(R)$. By Claim 6, for at least one agent $h \in N, f_{h}\left(\hat{R}_{1}, R_{-1}\right)<f_{h}(R)$. These imply

$$
E=\sum_{j \in N} f_{j}\left(\hat{R}_{1}, R_{-1}\right)<\sum_{j \in N} f_{j}(R)=E
$$

which is a contradiction. Therefore, for each $i \in N, f_{i}(R) \geq G_{i}(R)$. It thus follows from feasibility that for each $i \in N, f_{i}(R)=G_{i}(R)$.

Remark 2. Our theorem does not use the full force of strategy-proofness. In fact, Theorem 1 holds even if strategy-proofness is relaxed with "strategy-proofness for 
same tops" (Sakai and Wakayama, 2012). ${ }^{9}$ From this, we can easily obtain another characterization of the gradual uniform rule by replacing strategy-proofness with "peak-only." 10

Before concluding this section, we establish the tightness of Theorem 1. The uniform reallocation rule is an example of a rule satisfying all properties but not position-wise envy-freeness. The uniform rule is an example of a rule satisfying all properties but not individual rationality. The endowment rule (that always assigns to any agent his endowment) is an example of a rule satisfying all properties but not efficiency. The following example illustrates a rule satisfying all properties but not strategy-proofness.

Example 2. Let $n=3$ and $e=(1,2,1)$. Let $\hat{R} \in \mathscr{R}^{3}$ be such that $1 \hat{I}_{1} 3$ and $p(\hat{R})=(2,3,0)$, where $\hat{I}_{1}$ denote the indifference part of $\hat{R}_{1}$. Define $f^{*}$ as follows: for each $R \in \mathscr{R}^{3}$,

$$
f^{*}(R)= \begin{cases}(1,3,0) & \text { if } R=\hat{R} \\ G(R) & \text { otherwise }\end{cases}
$$

Then, $f^{*}$ is efficient, individually rational, and position-wise envy-free. However, $f^{*}$ is not strategy-proof. To see this, let $R_{1} \in \mathscr{R}$ be such that $p\left(R_{1}\right)=2$ and $3 P_{1} 1$. Then, $f^{*}\left(R_{1}, \hat{R}_{-1}\right)=G\left(R_{1}, \hat{R}_{-1}\right)=(2,2,0)$ and thus, $f_{1}^{*}\left(R_{1}, \hat{R}_{-1}\right)=2 \hat{P}_{1} 1=$ $f_{1}^{*}(\hat{R})$, a violation of strategy-proofness.

\footnotetext{
${ }^{9}$ The notion of "strategy-proofness for same tops" states that an agent cannot gain if he truthfully reports his peak.

${ }^{10}$ The notion of "peak-only" states that the amount assigned to agents depends only on their peaks.
} 


\section{References}

Barberà, S., M. O. Jackson, and A. Neme (1997). "Strategy-Proof Allotment Rules," Games and Economic Behavior 18, 1-21.

Benassy, J. P. (1982). The Economics of Market Disequilibrium, Academic Press, New York.

Bergantiños, G., J. Massó, and A. Neme (2015). "The Division Problem under Constraints," Games and Economic Behavior 89, 56-77.

Bonifacio, A. G. (2015). "Bribe-Proof Reallocation with Single-Peaked Preferences," Social Choice and Welfare 55, 617-638.

Ching, S. (1994). "An Alternative Characterization of the Uniform Rule," Social Choice and Welfare 11, 131-136.

Chun, Y. (2006). "The Separability Principle in Economies with Single-Peaked Preferences," Social Choice and Welfare 26, 239-253.

Foley, D. K. (1967). "Resource Allocation and the Public Sector," Yale Economic Essays 7, 45-98.

Klaus, B. (1998). Fair Allocation and Reallocation: An Axiomatic Study, Ph.D. thesis, Maastricht University, Maastricht, The Netherlands.

Klaus, B. (2001). "Uniform Allocation and Reallocation Revisited," Review of Economic Design 6, 85-98.

Klaus, B., H. Peters, and T. Storcken (1997). "Reallocation of an Infinitely Divisible Good," Economic Theory 10, 305-333.

Klaus, B., H. Peters, T. Storcken (1998a). "Strategy-Proof Division with SinglePeaked Preferences and Individual Endowments," Social Choice and Welfare 15, 297-311.

Klaus, B., H. Peters, T. Storcken (1998b). "Strategy-Proof Reallocation of an Infinitely Divisible Good," in P. L. Butzer, H. Th. Jongen, and W. Oberschelp (Eds.), Charlemagne and the Liberal Arts: 1200 Years of Civilization and Science in Europe, Volume2: Mathematical Arts, Brepols Verlag, Aachen, 455-470. 
Mizobuchi, H. and S. Serizawa (2006). "Maximal Domain for Strategy-Proof Rules in Allotment Economies," Social Choice and Welfare 27, 195-210.

Moreno, B. (2002). "Single-Peaked Preferences, Endowments and PopulationMonotonicity," Economics Letters 75, 87-95.

Sakai, T. and T. Wakayama (2012). "Strategy-Proofness, Tops-Only, and the Uniform Rule," Theory and Decision 72, 287-301.

Schmeidler, D. and K. Vind (1972). "Fair Net Trades," Econometrica 40, 637-642.

Serizawa, S. (2006). "Pairwise Strategy-Proofness and Self-Enforcing Manipulation," Social Choice and Welfare 26, 305-331.

Sönmez, T. (1994). "Consistency, Monotonicity, and the Uniform Rule," Economics Letters 46, 229-235.

Sprumont, Y. (1991). "The Division Problem with Single-Peaked Preferences: A Characterization of the Uniform Allocation Rule," Econometrica 59, 509-519.

Thomson, W. (1994a). "Resource-Monotonic Solutions to the Problem of Fair Division When Preferences are Single-Peaked," Social Choice and Welfare 11, $205-223$.

Thomson, W. (1994b). "Consistent Solutions to the Problem of Fair Division When Preferences are Single-Peaked," Journal of Economic Theory 63, 219-245.

Thomson, W. (1995). "Population-Monotonic Solutions to the Problem of Fair Division When Preferences are Single-Peaked," Economic Theory 5, 229-246.

Thomson, W. (1997). "The Replacement Principle in Economies with Single-Peaked Preferences," Journal of Economic Theory 76, 145-168.

Thomson, W. (2014). "Fully Allocating a Commodity among Agents with SinglePeaked Preferences," mimeo.

Wakayama, T. (2015). "Bribe-Proof Division of a Private Good," mineo. 